\title{
Two-Dimensional Lorentz-Weyl Anomaly and Gravitational Chern-Simons Theory
}

\author{
A.H. Chamseddine ${ }^{1}$ and J. Fröhlich ${ }^{2}$ \\ ${ }^{1}$ University of Zürich, Zürich, Switzerland \\ 2 ETH-Zürich, CH-8093 Zürich, Switzerland
}

Received December 30, 1991

\begin{abstract}
Two-dimensional chiral fermions and bosons, more generally conformal blocks of two-dimensional conformal field theories, exhibit Weyl-, Lorentz- and mixed Lorentz-Weyl anomalies. A novel way of computing these anomalies for a system of chiral bosons of arbitrary conformal spin $j$ is sketched. It is shown that the Lorentz- and mixed Lorentz-Weyl anomalies of these theories can be cancelled by the anomalies of a three-dimensional classical Chern-Simons action for the spin connection, expressed in terms of the dreibein field. Some tentative applications of this result to string theory are indicated.
\end{abstract}

There are two circles of problems in theoretical physics which lead us to reconsider some aspects of two-dimensional chiral anomalies. The first one concerns the theory of incompressible, chiral quantum fluids, in particular of two-dimensional electron fluids in a transverse, external magnetic field encountered in studies of the quantized Hall effect. The study of the dynamics of Hall fluids near the boundary of the system naturally leads one to consider $(1+1)$-dimensional abelian and nonabelian gauge anomalies and the associated abelian and non-abelian ChernSimons gauge theories in $2+1$ dimensions which describe bulk properties of two-dimensional, incompressible, chiral quantum fluids in the large-scale, lowfrequency limit $[1,2]$.

The second circle of problems concerns the anomalies, in particular the Lorentz- and Weyl anomalies, of two-dimensional (chiral) conformal field theory and the problem of constructing new string theories [3-5].

This note has grown out of studying these two circles of problems. It is quite likely that the following calculations and remarks are known to experts in the field. Nevertheless, we wish to submit the results of a series of exercises that we have performed to the attention of the interested reader.

Chiral fermions coupled to an external gravitational field in $(2+4 n)$ dimensions, $n=0,1,2, \ldots$, are known to exhibit Weyl- and Lorentz anomalies [6-8]. At the classical level, the action is invariant under Weyl transformations and Lorentz transformations of the local Lorentz frames. However, quantization of the fermions breaks these symmetries. The effective action, defined as the logarithm of the chiral-, or Weyl determinant, is not invariant under Weyl- and local Lorentz 
transformations. Actually, the breaking of Weyl invariance is common to all such theories, not just chiral ones.

We now recall some properties of two-dimensional chiral fermions. In twodimensional Euclidean space-time, it is fairly straightforward to calculate the Weyl determinant [8]. It is of the form $\exp (\mathscr{R}+i \mathscr{I})$. The real part, $\mathscr{R}$, only depnds on the metric, and, in the conformal gauge (where diffeomorphism invariance is gauge-fixed), it is given by the Liouville action. The imaginary part, $\mathscr{I}$ (the argument of the Euclidean Weyl determinant), explicitly depends on the spin connection, $\omega$, chosen on the two-dimensional space-time. In the conformal gauge, it really depends on two scalar fields, the Weyl- and the Lorentz modes of the zweibein $e_{a}^{j}$, (the other two modes of $e_{a}^{j}$ being frozen by fixing the conformal gauge). If the two-dimensional system contains equal numbers of holomorphic and antiholomorphic fermions the product of the corresponding Weyl determinants is real, and the Lorentz-anomalous terms $\pm i \mathscr{I}$ cancel each other. However, the Weyl anomaly remains. It could be cancelled by saturating the conformal anomaly of the system to become critical and then integrating over all metrics on the twodimensional space-time of the system.

Quantized chiral fermions coupled to external gauge fields are well known to exhibit gauge anomalies reflecting a breakdown of gauge invariance [9]. These anomalies were shown to be cancelled by those of a three-dimensional ChernSimons action with the same gauge group on a three-dimensional space-time whose boundary is the two-dimensional space-time of the fermionic system [9]. It is also known that the gauge anomalies of chiral fermions are identical to those of systems of free, chiral bosons on the same two-dimensional space-time, coupled to the same external gauge fields [10]. This is understood with the help of twodimensional chiral bosonization [11].

It is the purpose of this note to address the parallel problem of cancelling the Lorentz anomaly of two-dimensional chiral fermions, or of two-dimensional chiral bosons, by the Lorentz anomaly of an appropriate three-dimensional ChernSimons action. This action turns out to be a gravitational Chern-Simons action. However, unlike the gauge anomaly, the Lorentz anomaly mixes with the Weyl anomaly, and the cancellation mechanism is somewhat more delicate. What we show in this note is that the Lorentz- and mixed Lorentz-Weyl anomaly, but not the pure Weyl anomaly, of two-dimensional chiral fermions or bosons can be cancelled by a three-dimensional gravitational Chern-Simons action.

We choose two-dimensional space-time, $M_{2}$, to be Riemannian (or "Euclidean"). For simplicity, $M_{2}$ is chosen to be the plane or Riemann sphere. We note, however, that we can also choose $M_{2}$ to be the plane or a cylinder and to carry a Lorentzian metric; (where necessary, we shall indicate the appropriate modifications in the following arguments).

The two-dimensional Dirac-Weyl operator has the form

$$
D=i \sigma^{a} e_{a}^{j}\left(\partial_{j}+i \omega_{j}\right)
$$

where $\sigma^{1}$ and $\sigma^{2}$ are the two-dimensional Weyl matrices, $e_{a}^{j}$ is the inverse zweibein field, and $\omega_{j}$ is the $j^{\text {th }}$ component of the spin connection $\omega$ on $M_{2}$. There are two inequivalent representations of the Weyl matrices, $\sigma^{1}=1, \sigma^{2}= \pm i$, corresponding to holomorphic and anti-holomorphic fermions (or to left- or right 'moving 
fermions if $M_{2}$ is Lorentzian). The inverse zweibein fields $e_{1}^{j}(x), e_{2}^{j}(x), x \in M_{2}$, fix the metric $\left(g_{i j}\right)$, with inverse $\left(g^{i j}\right)$, on $M_{2}$ :

$$
g^{i j}(x)=\delta^{a b} e_{a}^{i}(x) e_{b}^{j}(x),
$$

where $\delta^{a b}$ is the Euclidean metric on the cotangent space to $M_{2}$ at $x$. [In the Lorentzian case, $\delta^{a b}$ is replaced by the flat Minkowski metric $\eta^{a b}$, and $g^{i j}$ is Lorentzian.]

Up to renormalization ambiguities described by local polynomials in the zweibein and inverse zweibein fields and derivatives thereof, the determinant of the Weyl operator $D$ defined in (1) is given by

$$
\operatorname{det} D=\exp \Gamma_{L / R}[e] \text {, }
$$

where

and

$$
\Gamma_{L / R}=\mathscr{R} \pm i \mathscr{I}
$$

$$
\begin{aligned}
\mathscr{R}(e)= & \frac{1}{192 \pi} \int_{M_{2}} d^{2} x \sqrt{g(x)} \int_{M_{2}} d^{2} y \sqrt{g(y)} R(x)\left(\Delta_{g}^{-1}\right)(x, y) R(y), \\
\mathscr{I}(e)= & \frac{-1}{192 \pi} \int_{M_{2}} d^{2} x \sqrt{g(x)} \int_{M_{2}} d^{2} y \sqrt{g(y)} R(x)\left(\Delta_{g}^{-1}\right)(x, y) \\
& \times \frac{1}{\sqrt{g(y)}} \partial_{i}\left(\sqrt{g(y)} g^{i j} \omega_{j}(y)\right),
\end{aligned}
$$

where $g(x)=\operatorname{det}\left(g_{i j}(x)\right),(\sqrt{g}$ would be replaced by $\sqrt{|g|}$, and $\mathscr{R}$ would be multiplied by $i \equiv \sqrt{-1}$ if the metric $g_{i j}$ were Lorentzian), $\Delta_{g}=-\frac{1}{\sqrt{g}} \partial_{i}\left(\sqrt{g} g^{i j} \partial_{j}\right)$ is the Laplace-Beltrami operator in the metric $\left(g_{i j}\right)$, and $R$ is the scalar curvature given by

$$
R=\frac{1}{\sqrt{g}} \varepsilon^{i j} \partial_{i} \omega_{j}
$$

The effective action for a system of $n_{L}$ holomorphic and $n_{R}$ anti-holomorphic fermions is then given by

$$
\Gamma[e]=\left(n_{R}+n_{L}\right) \mathscr{R}(e)+i\left(n_{R}-n_{L}\right) \mathscr{I}(e) .
$$

The imaginary part, $\mathscr{I}(e)$, of the Weyl determinant exhibits a Lorentz anomaly, since it explicitly depends on the spin connection $\omega$. Equation (8) shows that the Lorentz anomaly disappears if $n_{R}=n_{L}$, as expected.

In order to better understand the strucutre of $\Gamma[e]$, we introduce a parametrization of the zweibein $e_{j}^{a}$ in terms of a Weyl- and a Lorentz mode:

$$
e_{j}^{a}=e^{\frac{1}{2} \phi} \hat{e}_{j}^{b}\left(\delta_{b}^{a} \cos \frac{\sigma}{2}+\varepsilon_{b}^{a} \sin \frac{\sigma}{2}\right),
$$

where $\hat{e}_{j}^{a}$ is the zweibein corresponding to a fixed metric $\hat{g}_{i j}=\delta_{a b} \hat{e}_{i}^{a} \hat{e}_{j}^{b}$ on $M_{2}$, chosen conveniently depending on the topology of $M_{2}$. For example, if $M_{2}$ is the 
plane or a cylinder we choose "isothermal" coordinates in which $\hat{g}_{i j}$ is the flat Euclidean metric on $M_{2}$. [We note that if the metric on $M_{2}$ were chosen to be Lorentzian the trigonometric functions on the right-hand side of (9) would be replaced by the corresponding hyperbolic functions.] In terms of the variables in (9) the metric $g_{i j}$ is given by

$$
g_{i j}=\delta_{a b} e_{i}^{a} e_{j}^{b}=e^{\phi} \hat{g}_{i j}
$$

which shows that $\phi$ is the "Weyl mode." The dependence of the spin connection $\omega$ on the two fields $\phi$ and $\sigma$ is determined from the expression

$$
\omega_{i}=-2 \frac{1}{\sqrt{g}} \varepsilon^{j k}\left(\partial_{j} e_{k}^{a}\right) e_{i}^{b} \delta_{a b},
$$

where

$$
\omega_{i b}^{a}=\frac{1}{2} \omega_{i} \varepsilon_{b}^{a} .
$$

Substitution of Eq. (9) into Eq. (11) yields

$$
\omega_{i}=\hat{\omega}_{i}+\sqrt{g} \varepsilon_{i}^{j} \partial_{j} \phi-\partial_{i} \sigma .
$$

Equations (9) and (13) show that $\sigma$ is the "Lorentz mode;" $(\sigma / 2$ is an angle of rotation of the zweibein). From (7) and (13) we find that

$$
R=e^{-\phi}\left(\hat{R}+\Delta_{\hat{g}} \phi\right) .
$$

Combining Eqs. (5), (6) and (8) with (10), (13) and (14) we obtain that

$$
\begin{aligned}
\Gamma[e]= & \Gamma[\hat{e}]+\frac{1}{192 \pi} \int_{M_{2}} d^{2} x \sqrt{\hat{g}}\left\{\alpha\left(\phi \Delta_{\hat{g}} \phi+2 \phi \hat{R}\right)\right. \\
& \left.-i \beta\left(\sigma\left(\hat{R}+\Delta_{\hat{g}} \phi\right)-\hat{g}^{i j} \partial_{i} \phi \hat{\omega}_{j}\right)\right\},
\end{aligned}
$$

with $\alpha:=n_{R}+n_{L}, \beta:=n_{R}-n_{L}$. The term proportional to $\alpha$ on the right-hand side of (15) is the Liouville action, the first piece of the term proportional to $\beta$ is the Lorentz anomaly and the last two terms are the mixed Lorentz-Weyl anomaly.

Next, we wish to show that the same Lorentz-Weyl anomalies also appear in a theory of massless, chiral bosons. Let $\chi$ be a free, massless Bose field on $M_{2}$. Under local rotations of the zweibein $e_{j}^{a}$, as described in (9), $\chi$ is assumed to transform essentially like an angle:

$$
\chi \mapsto^{\sigma} \chi=\chi-k \sigma, \quad k=\frac{1}{2}\left(j-\frac{1}{2}\right),
$$

where $j$ is the conformal spin. Covariant derivatives of $\chi$ must therefore be defined by $\partial_{j} \chi-k \omega_{j}$.

It is convenient to choose a complex structure on $M_{2}$ compatible with its metric. We then introduce the differential operators $\partial \equiv \partial_{+}$and $\partial_{-} \equiv \bar{\partial}$. In local complex coordinates $z, \bar{z}$, on $M_{2}, \partial_{+}=\partial / \partial z$ and $\partial_{-}=\partial / \partial \bar{z}$. If the spin connection $\omega$ vanishes the field $\chi$ is chiral if

$$
\partial_{+} \chi=0, \quad \text { or } \partial_{-} \chi=0 .
$$

However, the constraints (17) are not invariant under local rotations of the zweibein $e_{j}^{a}$. A form of (17) invariant under local rotations of $e_{j}^{a}$ is given' by

$$
\partial_{ \pm} \chi-k \omega_{ \pm}=0,
$$


where, in the coordinates $z$ and $\bar{z}, \omega=\omega_{+} d z+\omega_{-} d \bar{z}$. If we intend to construct a theory of chiral bosons we must require that one of the constraints (18) be invariant under Weyl rescaling. In $(z, \bar{z})$-coordinates, $\omega_{ \pm} \mapsto^{\phi} \omega_{ \pm}=\omega_{ \pm} \pm i \partial_{ \pm} \phi$, by Eq. (13). Hence (18) is invariant under Weyl rescaling if

$$
\chi \mapsto^{\phi} \chi=\chi \pm i k \phi
$$

An action functional for the field $\chi$ is defined by

$$
\begin{aligned}
S_{j}(\chi, \omega)= & -\frac{i}{4 \pi} \int_{M_{2}} \partial_{+} \chi \partial_{-} \chi d z \wedge d \bar{z} \\
& +\frac{i k}{2 \pi} \int_{M_{2}}\left\{\omega_{-} \partial_{+} \chi-\omega_{+} \partial_{-} \chi\right\} d z \wedge d \bar{z} \\
& +\frac{i k^{2}}{4 \pi} \int_{M_{2}} \omega_{-} \omega_{+} d z \wedge d \bar{z}
\end{aligned}
$$

with $j=2 k+1 / 2$.

This definition of an action makes sense if $M_{2}$ is the punctured Riemann sphere and hence admits global coordinates. For more complicated topologies of $M_{2}, S(\chi, \omega)$ must be defined as a sum of terms corresponding to different coordinate patches; see e.g. [11]. Imposing the chiral constraint

$$
\partial_{-} \chi-k \omega_{-}=0
$$

with the transformation law

$$
\chi \mapsto^{\phi} \chi=\chi-i k \phi
$$

under Weyl rescaling, we find from (20) that

$$
\begin{aligned}
S_{j}(\chi, \omega)= & -\frac{i}{4 \pi} \int_{M_{2}} \partial_{+} \chi \partial_{-} \chi d z \wedge d \bar{z} \\
& +\frac{i k}{2 \pi} \int_{M_{2}} \omega_{-} \partial_{+} \chi d z \wedge d \bar{z}-\frac{i k^{2}}{4 \pi} \int_{M_{2}} \omega_{-} \omega_{+} d z \wedge d \bar{z} .
\end{aligned}
$$

The term

$$
-\frac{i k}{2 \pi} \int_{M_{2}} \omega_{+}\left(\partial_{-} \chi-k \omega_{-}\right) d z \wedge d \bar{z}
$$

is omitted from (23), since, by Eq. (21), it vanishes. Since ${ }^{\phi} \omega_{-}=\omega_{-}-i \partial_{-} \phi$ and thanks to Eqs. (22), (13) and (16), the constraint (21) is invariant under local rotations of the zweibein and Weyl rescaling, and hence (24) cannot contribute to the Lorentz- and Weyl anomalies.

We now determine how $S_{j}(\chi, \omega)$ transforms under local rotations of the zweibein and Weyl rescaling. We set

$$
\chi=\hat{\chi}-k \sigma-i k \phi,
$$

and

$$
\omega_{ \pm}=\hat{\omega}_{ \pm}-\partial_{ \pm} \sigma \pm i \partial_{ \pm} \phi
$$


Substituting expressions (25) and (26) into Eq. (23) for the action functional, we find, after a somewhat lengthy, but straightforward calculation, that

$$
\begin{aligned}
S_{j}(\chi, \omega)= & S_{j}(\hat{\chi}, \hat{\omega})-\frac{i k^{2}}{2 \pi} \int_{M_{2}}\left\{\partial_{+} \phi \partial_{-} \phi+i\left(\partial_{+} \phi \hat{\omega}_{-}-\partial_{-} \phi \hat{\omega}_{+}\right)\right\} d z \wedge d \bar{z} \\
& +\frac{k^{2}}{4 \pi} \int_{M_{2}}\left(\partial_{-} \phi \hat{\omega}_{+}+\partial_{+} \phi \hat{\omega}_{-}\right) d z \wedge d \bar{z}-\frac{k^{2}}{2 \pi} \int \partial_{+} \sigma \partial_{-} \phi d z \wedge d \bar{z} \\
& -\frac{i k^{2}}{4 \pi} \int\left\{\hat{\omega}_{-} \partial_{+} \sigma-\hat{\omega}_{+} \partial_{-} \sigma\right\} d z \wedge d \bar{z} .
\end{aligned}
$$

Using that

$$
\begin{aligned}
\partial_{+} \partial_{-} & =-\frac{1}{4} \Delta_{\hat{g}}, \partial_{+} \hat{\omega}_{-}-\partial_{-} \hat{\omega}_{+}=\frac{i}{2} \hat{R}, \quad \text { and } \\
d z \wedge d \bar{z} & =-2 i \sqrt{\hat{g}} d x \wedge d y,
\end{aligned}
$$

we find that the anomaly terms on the right-hand side of Eq. (27) are identical to those of the action $-48 k^{2} \Gamma_{L}[e]$. This is, of course, not an accident. Let us consider the formal functional integral

$$
e^{\Gamma_{j}[e]}=\int e^{S_{j}(\chi, \omega)} \delta\left(\partial_{-} \chi-k \omega_{-}\right) \mathscr{D}_{g} \chi,
$$

with $j=2 k+\frac{1}{2}$. By using quadratic completion to rewrite $S_{j}(\chi, \omega)$ and translating the integration- $(\chi-)$ variables we find that

$$
e^{\Gamma_{j}[e]}=e^{\Gamma_{1 / 2}[e]} \exp \left[-\frac{i k^{2}}{4 \pi} \int_{M_{2}}\left(\omega_{+} \omega_{-}+\partial_{+} \omega_{-}\left[\left(\partial_{+} \partial_{-}\right)^{-1} \partial_{+} \omega_{-}\right]\right) d z \wedge d \bar{z}\right] \text {, }
$$

where

$$
e^{\Gamma_{1 / 2}[e]}=\int e^{S_{1 / 2}(\chi, 0)} \delta\left(\partial_{-} \chi\right) \mathscr{D}_{g} \chi
$$

Of course, the right-hand sides of (29) and (31) are ill-defined, as written. However, $\exp \Gamma_{1 / 2}[e]$ can be defined rigorously by interpreting this functional as the partition function of a chiral boson system with conformal $\operatorname{spin} j=1 / 2$, i.e., $k=0$, which is known to be equivalent to a free Weyl fermion, [11]. Thus

$$
\Gamma_{1 / 2}[e]=\Gamma_{L}[e] \text {, }
$$

where $\Gamma_{L}[e]$ has been defined in Eqs. (4), (5), (6). It might, a priori, seem puzzling that the functional integral on the right-hand side of (31) depends on the zweibein $e_{i}^{a}$. On a formal level, this dependence is, however, explained in terms of the dependence of $\mathscr{D}_{g} \chi$ on $g$, and the dependence on $\omega$ comes from the circumstance that the system is chiral.

By Eq. (30), our interpretation (32) of $\Gamma_{1 / 2}[e]$ provides a precise definition of $\Gamma_{j}[e]$, for arbitrary values of $j$.

Using Eqs. (4), (5), (6) and (28), we find that

$$
\Gamma_{L}[e]=\frac{i}{192 \pi} \int_{M_{2}}\left(\omega_{+} \omega_{-}+\partial_{+} \omega_{-}\left[\left(\partial_{+} \partial_{-}\right)^{-1} \partial_{+} \omega_{-}\right]\right) d z \wedge d \bar{z},
$$


and hence from (30)

$$
\Gamma_{j}[e]=-\left(48 k^{2}-1\right) \Gamma_{L}[e]=c_{j} \Gamma_{L}[e],
$$

where

$$
c_{j}=-2\left(6 j^{2}-6 j+1\right)
$$

is the Virasoro central charge of the system.

Since these results hold for arbitrary values of $j$, we conclude that the effective action derived from a conformal block of a conformal field theory with Virasoro central charge $c$ must be given by

$$
\Gamma_{c}[e]=c \Gamma_{L}[e] .
$$

Of course, an analogous analysis applies to theories where the roles of " + " and "- " and " $L$ " and " $R$ " are interchanged.

Having found the expression for the Lorentz-Weyl anomaly of general chiral conformal systems (including chiral fermions and bosons) we next address the problem of cancelling the Lorentz- and the mixed Lorentz-Weyl anomalies of these systems by those of a three-dimensional "topological" action. The solution to this problem will represent the main result of this note.

In analogy with the cancellation of the two-dimensional chiral gauge anomaly by that of a three-dimensional topological Chern-Simons gauge theory with the same gauge group, we propose to construct a suitable three-dimensional gravitational Chern-Simons action cancelling the two-dimensional Lorentz- and mixed Lorentz-Weyl anomaly. We consider a surface $M_{2}$ which is the boundary of a three-dimensional Riemannian (or Lorentzian) manifold $M_{3}$. The action we are looking for is defined on $M_{3}$ and must be invariant under local rotations of the dreibein and three-dimensional Weyl rescaling, except for a winding-number term and boundary terms localized on $M_{2}=\partial M_{3}$.

There are two possible gravitational Chern-Simons actions. The first one is based on the gauge group $\operatorname{ISO}(3)$ (or $\operatorname{ISO}(2,1)$ ) and is identical, up to a boundary term, to the first order formalism for the Einstein-Hilbert action. This action is, however, known not to be Weyl-invariant, as one easily checks. The second action is the Chern-Simons action for the spin connection based on the gauge group $\operatorname{SU}(2)$ (or $\operatorname{SL}(2, \mathbb{R})$ ). We shall show that this action is invariant under local rotations of the dreibein and Weyl-invariant, except for boundary terms.

Let

$$
\omega=\frac{1}{2} \omega^{A B} \gamma_{A B}
$$

denote the three-dimensional spin connection, with $\gamma_{A B}=\frac{1}{2}\left[\gamma_{A}, \gamma_{B}\right],\left\{\gamma_{A}, \gamma_{B}\right\}=2 \delta_{A B}$, (or $\left\{\gamma_{A}, \gamma_{B}\right\}=2 \eta_{A B}$ if $M_{3}$ is Lorentzian), $A, B=0,1,2$. The dreibein one-form is denoted by

$$
e=e^{A} \gamma_{A}
$$

By Cartan's first structure equation, the zero-torsion constraint is expressed by the equation

$$
d e^{A}+\omega^{A}{ }_{B} e^{B}=0 .
$$


The action we are looking for is given by ${ }^{1}$

$$
I \equiv I[e, \omega, \lambda]=\frac{i}{4 \pi} \int_{M_{3}}\left\{\operatorname{tr}\left(\omega d \omega+\frac{2}{3} \omega^{3}\right)+\lambda_{A}\left(d e^{A}+\omega^{A}{ }_{B} e^{B}\right)\right\},
$$

where $\lambda_{A}$ is a one-form Lagrange-multiplier, and we have dropped the symbol " $\wedge$ " for the wedge product in our notation. The role of $\lambda_{A}$ is to impose the zero-torsion constraint (38) through its equation of motion. Actions analogous to $I[e, \omega, \lambda]$ have been considered before in all odd dimensions $[12,13]$.

We can solve Eq. (38) for $\omega$. The result is

$$
\omega_{\mu B C}(e)=\frac{1}{2} e_{\mu}^{A}\left(\Omega_{A B C}-\Omega_{B C A}+\Omega_{C A B}\right),
$$

where

$$
\Omega_{A B}{ }^{C}=e_{A}^{\mu} e_{B}^{v}\left(\partial_{\mu} e_{v}^{C}-\partial_{v} e_{\mu}^{C}\right) .
$$

Substituting (40) into (39) we can eliminate $\omega$ and find

$$
I[e]=\frac{i}{4 \pi} \int_{M_{3}}\left\{\omega^{A}{ }_{B}(e) d \omega^{B}{ }_{A}(e)+\frac{2}{3} \omega^{A}{ }_{B}(e) \omega^{B}{ }_{C}(e) \omega^{C}{ }_{A}(e)\right\} .
$$

This action is referred to as the "spin-connection Chern-Simons action." ${ }^{2}$ It is related to the following "Levi-Cività-connection Chern-Simons action"

$$
I^{\prime}\left[g_{\mu \nu}\right]=\frac{i}{4 \pi} \int_{M_{3}}\left\{\Gamma_{\mu \kappa}^{\sigma} \partial_{\nu} \Gamma_{\rho \sigma}^{\kappa}+\frac{2}{3} \Gamma_{\mu \kappa}^{\sigma} \Gamma_{\nu \lambda}^{\kappa} \Gamma_{\rho \sigma}^{\lambda}\right\},
$$

but $I$ and $I^{\prime}$ are not identical. The reason why we choose $I$ rather than $I^{\prime}$ is that $I$ displays a Lorentz anomaly at the boundary, but diffeomorphism invariance is preserved. Instead, $I^{\prime}$ is obviously invariant under local rotations of the dreibein, since it only depends on the metric $g_{\mu \nu}$, but it breaks diffeomorphism invariance at the boundary. It could be used to cancel the diffeomorphism anomaly of chiral theories on $M_{2}$.

The relation between $I$ and $I^{\prime}$ is given by

$$
\begin{aligned}
I[e]= & I^{\prime}\left[g_{\mu \nu}\right]+\frac{i}{12 \pi} \int_{M_{3}}\left(e^{-1} d e\right)_{A}{ }^{B}\left(e^{-1} d e\right)_{B}{ }^{C}\left(e^{-1} d e\right)_{C}{ }^{A} \\
& +\frac{i}{4 \pi} \int_{\partial M_{3}} \varepsilon^{i j} \partial_{i} e_{\kappa}^{a} \Gamma_{j \lambda}^{\kappa} e_{a}^{\lambda} d^{2} x
\end{aligned}
$$

where $\left(e^{-1} d e\right)_{A}^{B}=e_{A}^{\kappa} d e_{\kappa}^{B}$, and we have used the identity

$$
\omega_{\mu B}^{A}=-\partial_{\mu} e_{\nu}^{A} e_{B}^{v}+\Gamma_{\mu \nu}^{\rho} e_{\rho}^{A} e_{B}^{v} .
$$

In (44), the Lorentz index $a$ and the spatial indices $i$ and $j$ range over 0 and 1 . On the boundary $\partial M_{3}=M_{2}$, we require $e^{2}$ to be normal to $M_{2}$, and we choose local

\footnotetext{
${ }^{1}$ In this action the dreibein field is not a gauge field, but related to the metric of the manifold. The energy-momentum tensor does not vanish, and the theory is not topological

2 The action (42) has been shown recently to be needed as a counterterm in the renormalization of Chern-Simons theories [14]
} 
coordinates $\left(x^{0}, x^{1}, x^{2}\right)$ near $\partial M_{3}$ such that $x^{2}$ is transversal to $\partial M_{3}$, with

$$
\left.e_{i}^{2}\right|_{\partial M_{3}}=0, \text { for } i=0,1 .
$$

More details are presented in the Appendix.

Next, we wish to determine the transformation properties of the action $I[e]$ under local rotations of the dreibein $e_{\mu}^{A}$ preserving the constraint (46) and under Weyl transformations:

$$
e_{\mu}^{A} \mapsto e^{\frac{1}{2} \phi}\left[h^{-1} e_{\mu} h\right]^{A},
$$

where $h$ is $\mathrm{SU}(2)$-valued (or $\mathrm{SL}(2, \mathbb{R})$-valued if $M_{3}$ is Lorentzian), and, at the boundary $\partial M_{3}, e^{2}$ is invariant under $h$. It follows from Eq. (40) that the transformation (47) acts on the spin connection $\omega$ according to

$$
\omega_{\mu}{ }_{B}^{A} \mapsto\left(h^{-1} \omega_{\mu} h+h^{-1} \partial_{\mu} h\right)^{A}{ }_{B}+\frac{1}{2}\left(e_{\mu}^{A} e_{B}^{v}-e_{\mu B} e^{\nu A}\right) \partial_{\nu} \phi .
$$

Finding the transformation properties of the action (42) under the transformation (48) is quite difficult. It is simpler to proceed in two steps: Under local rotations

$$
e \mapsto^{h} e=h^{-1} e h
$$

the action (42) transforms in a well known way,

$$
I\left[{ }^{h} e\right]=I[e]+\frac{i}{4 \pi} \int_{\partial M_{3}} \operatorname{tr}\left(\omega(e) d h h^{-1}\right)-\frac{i}{12 \pi} \int_{M_{3}} \operatorname{tr}\left(h^{-1} d h\right)^{3} .
$$

If, after the transformation (49), we apply a Weyl transformation

$$
e_{\mu}^{A} \mapsto{ }^{\phi} e_{\mu}^{A}=e^{\frac{1}{2} \phi} e_{\mu}^{A}
$$

to the right-hand side of (50) we shall have found the transformation of the action $I[e]$ defined in (42) under the combined transformation (47) of $e_{\mu}^{A}$. The lengthy part of the calculation is to evaluate $I\left[e^{\frac{1}{2} \phi} e_{\mu}^{A}\right]$. To this end it is useful to use identity (44) between the actions $I$ and $I^{\prime}$ and to first evaluate $I^{\prime}\left[e^{\phi} g_{\mu \nu}\right]$. After some lengthy algebra sketched in the Appendix we find that

$$
\begin{aligned}
I^{\prime}\left[e^{\phi} g_{\mu \nu}\right] & =I^{\prime}\left[g_{\mu \nu}\right]+\frac{i}{8 \pi} \int_{\partial M_{3}} \varepsilon^{i j}\left(\Gamma_{\rho i}^{\gamma} g^{\rho \sigma} g_{j \gamma} \partial_{\sigma} \phi\right), \\
\frac{i}{12 \pi} \int_{M_{3}}\left({ }^{\phi} e^{-1} d^{\phi} e\right)^{3} & =\frac{i}{12 \pi} \int_{M_{3}}\left(e^{-1} d e\right)^{3}-\frac{i}{8 \pi} \int_{\partial M_{3}} \varepsilon^{i j} \phi \partial_{i} e_{A}^{\kappa} \partial_{j} e_{\kappa}^{A} .
\end{aligned}
$$

Plugging these equations into the right-hand side of identity (44) we find the simple result

$$
I\left[{ }^{\phi} e\right]=I[e]+\frac{i}{4 \pi} \int_{\partial M_{3}} \varepsilon^{i j}\left(\partial_{i} e_{j}^{A}\right) e_{A}^{k} \partial_{k} \phi .
$$

Combining Eqs. (50) and (53) we find that

$$
\begin{aligned}
& I\left[e^{\frac{1}{2} \phi}\left(h^{-1} e h\right)_{\mu}^{A}\right]=I\left[e_{\mu}^{A}\right]+\frac{i}{4 \pi} \int_{\partial M_{3}} \varepsilon^{i j}\left[\omega_{i B}^{A}\right. \\
&\left.+\frac{1}{2}\left(e_{i}^{A} e_{B}^{k}-e_{i B} e^{k A}\right) \partial_{k} \phi\right]\left(\left(\partial_{j} h\right) h^{-1}\right)_{A}^{B} \\
&+\frac{i}{4 \pi} \int_{\partial M_{3}} \varepsilon^{i j} \partial_{i} e_{j}^{A} e_{A}^{k} \partial_{k} \phi-\frac{i}{12 \pi} \int_{M_{3}} \operatorname{tr}\left(h^{-1} d h\right)^{3} .
\end{aligned}
$$


In order to make contact with the two-dimensional Lorentz-Weyl anomaly, we recall that, at the boundary of $M_{3}, e_{\mu}^{A}$ is constrained by Eq. (46), and the spin connection $\omega_{i B}^{A}$, restricted to $\partial M_{3}$, is the spin connection of the surface $\partial M_{3}=M_{2}$, i.e.,

$$
\left.\omega_{i B}^{A}\left(e_{\mu}^{A}\right)\right|_{\partial M_{3}}=\omega_{i b}^{a}\left(e_{j}^{a}\right),
$$

for $A=a=0,1, B=b=1,0$, and $i, j=0,1$, and $\left.\omega_{i B}^{A}\right|_{\partial M_{3}}=0$, for $A$ or $B=2$. The indices $a$ and $b$ label components of vectors in the (co-)tangent spaces to $M_{2}$.

We have already remarked above that

$$
\left((d h) h^{-1}\right)_{A}{ }^{B} \mapsto\left((d h) h^{-1}\right)_{a}{ }^{b}=\frac{1}{2} d \sigma \varepsilon_{a}{ }^{b},
$$

at the boundary, $M_{2}$, of $M_{3}$, so that the constraint (46) is invariant under local rotations of the dreibein satisfying (56). With Eqs. (46), (55) and (56) taken into account, Eq. (54) simplifies to

$$
\begin{aligned}
I\left[e^{\frac{1}{2} \phi}\left(h^{-1} e h\right)_{\mu}^{A}\right]= & I\left[e_{\mu}^{A}\right]+\frac{i}{8 \pi} \int_{\partial M_{3}} \varepsilon^{i j}\left(\omega_{i}-\sqrt{g} \varepsilon_{i k} \partial^{k} \phi\right) \partial_{j} \sigma \\
& +\frac{i}{4 \pi} \int_{\partial M_{3}} \varepsilon^{i j} \partial_{i} e_{j}^{a} e_{a}^{k} \partial_{k} \phi \\
& -\frac{i}{12 \pi} \int_{M_{3}} \operatorname{tr}\left(h^{-1} d h\right)^{3},
\end{aligned}
$$

where $\omega_{i b}^{a}=\frac{1}{2} \omega_{i} \varepsilon_{b}^{a}$. The third term on the right-hand side of Eq. (57) can be rewritten, using Eq. (11), as

$$
-\frac{i}{8 \pi} \int_{\partial M_{3}} \sqrt{g} g^{i j} \omega_{i} \partial_{j} \phi
$$

so that, after an integration by parts, Eq. (57) becomes

$$
\begin{aligned}
I\left[e^{\frac{1}{2} \phi}\left(h^{-1} e h\right)_{\mu}^{A}\right]= & I\left[e_{\mu}^{A}\right]+\frac{i}{8 \pi} \int_{\partial M_{3}}\left(\varepsilon^{i j} \partial_{i} \omega_{j}+\sqrt{g} \Delta_{g} \phi\right) \sigma \\
& -\frac{i}{8 \pi} \int_{\partial M_{3}} \sqrt{g} g^{i j} \omega_{i} \partial_{j} \phi-\frac{i}{12 \pi} \int_{M_{3}} \operatorname{tr}\left(h^{-1} d h\right)^{3} .
\end{aligned}
$$

Setting $g_{\mu \nu}=\hat{g}_{\mu \nu}, e_{\mu}^{A}=\hat{e}_{\mu}^{A}$ and $\omega_{\mu}=\hat{\omega}_{\mu}$, with $\left.\hat{e}_{\mu}^{A}\right|_{\partial M_{3}}=\hat{e}_{i}^{a}$, for $A=a$ $=0,1, \mu=i=0,1$ and $\left.\hat{\omega}_{\mu}\right|_{\partial M_{3}}=\hat{\omega}_{i}$, for $\mu=i=0$, 1 , we find that, apart from the last term on the right-hand side of (59), the anomaly of the gravitational ChernSimons action $I[e]$ cancels the Lorentz- and the mixed Lorentz-Weyl anomaly of $24 \Gamma_{L}[e]$. This is seen by comparing Eq. (59) to Eq. (15), (with $\alpha=-\beta=1$ ), or to (27), (with $k^{2}=\frac{1}{48}$ ).

Next, we consider the last term on the right-hand side of (59). Let $h$ be an arbitrary SU(2)-valued transformation on $M_{3}$ with the property that $h(x)$ tends continuously to the identity, $\mathbb{1}$, as $x \rightarrow \partial M_{3}=M_{2}$, and that the support of $h(x)-\mathbb{1}$ 
in $M_{3}$ is homeomorphic to a ball. Such transformations fall into different homotopy classes labelled by their winding numbers, $n(h)$, where

$$
2 \pi n(h)=\frac{1}{12 \pi} \int_{M_{3}} \operatorname{tr}\left(h^{-1} d h\right)^{3} .
$$

We conclude that, for every level $N=1,2,3, \ldots$, the functionals

$$
e^{N I[e]+24 N \Gamma_{L}[e]}
$$

and

$$
e^{-N I[e]+24 N \Gamma_{R}[e]}
$$

are free of Lorentz-(SU(2)-)anomalies and mixed Lorentz-Weyl anomalies. By Eqs. (5) and (15), these functionals only exhibit a Weyl anomaly given by

$$
\exp \left[+\frac{N}{8 \pi} \int_{M_{2}} d^{2} x \sqrt{\hat{g}}\left(\phi \Delta_{\hat{g}} \phi+2 \phi \hat{R}\right)\right] \text {. }
$$

Had we chosen $M_{3}$ and $M_{2}$ to carry Lorentzian, instead of Riemannian, metrics then the term $\int_{M_{3}} \operatorname{tr}\left(h^{-1} d h\right)^{3}$ would vanish, because $h$ would then be $\operatorname{SL}(2, \mathbb{R})$ valued, instead of SU(2)-valued. There is then, a priori, no quantization of the level $N$.

We feel that the main results, Eqs. (59)-(63), of this note provide another glimpse at a three-dimensional aspect of string theory, relating it to Chern-Simons gauge theories and gravitational Chern-Simons theories. However, if one really wishes to apply our results to string theory one must "quantize" the three-dimensional Chern-Simons theories. This is fairly well understood for Chern-Simons gauge theories [15], but less well understood for the gravitational Chern-Simons theory considered in this note. One would expect that if $M_{3}$, and hence $M_{2}$, carry Riemannian metrics functional integration of exp $-I[e]$ over $\left\{e_{\mu}^{A}\right\}$, subject to the boundary conditions (46), will yield conformal blocks cancelling the Lorentz- and mixed Lorentz-Weyl anomalies of $\exp 24 \Gamma_{L}[e]$ and contributing to the Weyl anomaly (63), in accordance with (33); (this contribution can be traced back to the dependence of the formal volume element $\mathscr{D}_{g} e_{\mu}^{A}$ on the Weyl mode, [16]).

It is tempting to speculate that the conformal blocks of all conformal field theories can be obtained from three-dimensional Chern-Simons theories; see [17] for related conjectures. If true such a result would be useful in finding a foundation for string theory in three-dimensional topological field theory. This would require understanding the dependence of the conformal blocks obtained from the three-dimensional theories, (e.g. from the integral of exp $-I[e]$ over $\left.\left\{e_{\mu}^{A}\right\}\right)$, on the topology of $M_{3}$ and on Teichmüller parameters related to $M_{2}=\partial M_{3}$ when $M_{2}$ is a higher-genus Riemann surface. A three-dimensional foundation for string theory might permit one to construct tunnelling amplitudes between different string vacua and to think about "sums over world sheet topologies" in a novel way. 


\section{Appendix}

In this appendix we shall derive some basic identities for the gravitational ChernSimons action which are used in this paper.

The first identity is a relation between two Chern-Simons actions, one being a function of the spin-connection and the other one a function of the Christoffel symbols. Let

$$
\begin{aligned}
I & =\int\left(\omega_{A}^{B}(e) d \omega_{B}{ }^{A}(e)+\frac{2}{3} \omega_{A}^{B}(e) \omega_{B}{ }^{C}(e) \omega_{C}{ }^{A}(e)\right), \\
I^{\prime} & =\int \varepsilon^{\mu \nu \rho}\left(\Gamma_{\mu \kappa}^{\sigma} \partial_{\nu} \Gamma_{\rho \sigma}^{\kappa}+\frac{2}{3} \Gamma_{\mu \kappa}^{\sigma} \Gamma_{\nu \lambda}^{\kappa} \Gamma_{\rho \sigma}^{\lambda}\right),
\end{aligned}
$$

where $\omega_{\mu A}{ }^{B}(e)$ is given in Eq. (12) and $\Gamma_{\mu \nu}^{\rho}$ is

$$
\Gamma_{\mu \nu}^{\rho}=\frac{1}{2} g^{\rho \sigma}\left(g_{\mu \sigma, v}+g_{v \sigma, \rho}-g_{\mu \nu, \sigma}\right)
$$

Using Eq. (12) it can be easily shown that the combined covariant derivative of the dreibein $e_{\mu}^{A}$ with respect to coordinate and Lorentz transformations vanishes

$$
\nabla_{\mu} e_{v}^{A} \equiv \partial_{\mu} e_{\nu}^{A}+\omega_{\mu B}^{A} e_{v}^{B}-\Gamma_{\mu \nu}^{\rho} e_{\rho}^{A}=0
$$

This can be solved for $\omega_{\rho}{ }^{A}$ to give

$$
\omega_{\mu}^{A}{ }_{B}(e)=\Gamma_{\mu \nu}^{\rho} e_{\rho}^{A}-\partial_{\mu} e_{\nu}^{A} e_{B}^{v} .
$$

Substituting Eq. (A.5) into Eq. (A.1) would immediately change the action $I$ into $I^{\prime}$, plus additional terms.

The exact form is found from the expansion of the two terms in Eq. (A.1). The first term gives

$$
\begin{aligned}
\varepsilon^{\mu \nu \rho} \omega_{\mu}^{A}{ }_{B} \partial_{\nu} \omega_{\rho}^{B}{ }_{A}= & \varepsilon^{\mu \nu \rho}\left[\Gamma_{\mu \kappa}^{\sigma} \partial_{\nu} \Gamma_{\rho \sigma}^{\kappa}+\Gamma_{\rho \kappa}^{\sigma} \Gamma_{\rho \sigma}^{\delta} \partial_{\nu} e_{\delta}^{B} e_{B}^{\kappa}\right. \\
& +\Gamma_{\mu \kappa}^{\sigma} \Gamma_{\rho \lambda}^{\kappa} e_{\sigma}^{A} \partial_{\nu} e_{A}^{\lambda}-\Gamma_{\mu \kappa}^{\sigma} e_{\sigma}^{A} e_{B}^{\kappa} \partial_{\rho} e_{\delta}^{B} \partial_{\nu} e_{A}^{\delta} \\
& -\partial_{\mu} e_{\kappa}^{A} \partial_{\nu} \Gamma_{\rho \lambda}^{\kappa} e_{A}^{\lambda}-\partial_{\mu} e_{\kappa}^{A} \Gamma_{\rho \lambda}^{\delta} e_{B}^{\kappa} \partial_{\nu} e_{\delta}^{B} e_{A}^{\lambda} \\
& \left.+\partial_{\mu} e_{\kappa}^{A} e_{B}^{\kappa} \partial_{\rho} e_{\delta}^{B} \partial_{\nu} e_{A}^{\delta}\right] .
\end{aligned}
$$

The second term is simpler and gives

$$
\begin{aligned}
\frac{2}{3} \varepsilon^{\mu \nu \rho} \omega_{\mu}{ }_{B}{ }_{B} \omega_{\nu}{ }^{B}{ }_{C} \omega_{\rho}{ }^{C}{ }_{A}= & 2 \varepsilon^{\mu \nu \rho}\left(\frac{1}{3} \Gamma_{\mu \kappa}^{\sigma} \Gamma_{v \lambda}^{\kappa} \Gamma_{\rho \sigma}^{\lambda}-\Gamma_{\mu \kappa}^{\sigma} \Gamma_{\nu \lambda}^{\kappa} \partial_{\rho} e_{\sigma}^{C} e_{C}^{\lambda}\right. \\
& +\Gamma_{\mu \kappa}^{\sigma} \partial_{\nu} e_{\delta}^{B} \partial_{\rho} e_{\sigma}^{C} e_{C}^{\delta} e_{B}^{\kappa} \\
& \left.-\frac{1}{3}\left(e^{-1} d e\right)_{B}{ }^{A}\left(e^{-1} d e\right)_{A}{ }^{C}\left(e^{-1} d e\right)_{C}{ }^{B}\right)
\end{aligned}
$$

By grouping similar terms, and reordering dummy indices, one can show that the terms $\Gamma \Gamma e^{-1} \partial e$ cancel among themselves. The terms $\Gamma e^{-1} \partial e e^{-1} \partial e$ would partially cancel with the remainder combining with $\partial \Gamma e^{-1} \partial e$ to form a total derivative

$$
\varepsilon^{\mu \nu \rho} \partial_{\mu}\left(\partial_{\nu} e_{\kappa}^{A} \Gamma_{\rho \lambda}^{\kappa} e_{A}^{\lambda}\right) .
$$

The final relation is then the identity in Eq. (16). 
The Weyl transformation of the action $I$, could be obtained by first deriving the Weyl transformation of the action $I^{\prime}$ and then using the identity in Eq. (16).

Under the Weyl scaling

$$
e_{\mu}^{A} \rightarrow e^{\frac{1}{2} \phi} e_{\mu}^{A}
$$

the Christoffel connection and the spin connection transform as

$$
\begin{gathered}
\Gamma_{\mu \nu}^{\rho} \rightarrow \Gamma_{\mu \nu}^{\rho}+\frac{1}{2}\left(\delta_{\mu}^{\rho} \partial_{\nu} \phi+\delta_{\nu}^{\rho} \partial_{\mu} \phi-g_{\mu \nu} g^{\rho \sigma} \partial_{\sigma} \phi\right), \\
\omega_{\mu}{ }_{B} \rightarrow \omega_{\mu}{ }^{A}{ }_{B}+\frac{1}{2}\left(e_{\mu}^{A} e_{B}^{v}-e_{\mu B} e^{A v}\right) \partial_{\nu} \phi .
\end{gathered}
$$

Substituting the transformation (A.10) into the action $I^{\prime}$, one finds that the first term transforms to

$$
\begin{aligned}
\varepsilon^{\lambda \mu \nu} \Gamma_{\lambda \gamma}^{\rho} \partial_{\mu} \Gamma_{\rho \nu}^{\gamma} \rightarrow & \varepsilon^{\lambda \mu \nu}\left(\Gamma_{\lambda \gamma}^{\rho} \partial_{\mu} \Gamma_{\rho \nu}^{\gamma}-\frac{1}{2} \Gamma_{\lambda \gamma}^{\rho} \partial_{\mu}\left(g^{\gamma \sigma} g_{\rho \nu} \partial_{\sigma} \phi\right)\right. \\
& -\frac{1}{2} g^{\rho \delta} g_{\lambda \gamma} \partial_{\mu} \Gamma_{\rho \nu}^{\gamma} \partial_{\delta} \phi+\frac{1}{4} g_{\lambda \gamma} \partial_{\mu} g^{\nu \sigma} \partial_{\nu} \phi \partial_{\sigma} \phi \\
& \left.+\frac{1}{4} g^{\rho \delta} \partial_{\mu} g_{\rho \nu} \partial_{\delta} \phi \partial_{\lambda} \phi\right)
\end{aligned}
$$

The second term transforms to a large number of terms which after some cancellations reduce to

$$
\begin{aligned}
\varepsilon^{\lambda \mu \nu} \Gamma_{\lambda \gamma}^{\rho} \Gamma_{\mu \tau}^{\gamma} \Gamma_{v \rho}^{\tau} \rightarrow & \varepsilon^{\lambda \mu \nu}\left[\Gamma_{\lambda \gamma}^{\rho} \Gamma_{\mu \tau}^{\gamma} \Gamma_{v \rho}^{\tau}-\frac{3}{4} \Gamma_{\lambda \gamma}^{\rho} g_{\mu \rho} g^{\nu \delta} \partial_{\delta} \phi \partial_{\nu} \phi\right. \\
& \left.-\frac{3}{2} \Gamma_{\mu \tau}^{\gamma} g^{\rho \sigma} g_{\lambda \gamma} \Gamma_{v \rho}^{\tau} \partial_{\sigma} \phi\right] .
\end{aligned}
$$

Combining Eqs. (A.12) and (A.13), one finds that the terms linear in $\partial \phi$ give a term proportional to the curvature which vanishes by the cyclic identity, as well as the total derivative

$$
\frac{1}{2} \partial_{\mu}\left(\Gamma_{\rho \nu}^{\gamma} g^{\rho \sigma} g_{\lambda \gamma} \partial_{\sigma} \phi\right)
$$

The quadratic terms in $\partial \phi$ can be easily shown to vanish.

We then have to identify

$$
I^{\prime}\left(e^{\phi} g_{\mu \nu}\right)=I^{\prime}\left(g_{\mu \nu}\right)+\frac{1}{2} \int_{\partial M_{3}} \varepsilon^{i j}\left(\Gamma_{\rho i}^{\gamma} g^{\rho k} g_{j \gamma} \partial_{k} \phi\right) .
$$

This shows that the Chern-Simons action as a function of the spin-connection is invariant under Weyl scaling up to a boundary term. It can also be seen from the identity in Eq. (16) that $I^{\prime}$ is invariant under general coordinate transformations inside $M_{3}$ but not on the boundary, unlike $I$ which is obviously invariant. 
The Weyl-transformation of the "winding-like" term can be evaluated to be

$$
\begin{aligned}
\frac{1}{3} \int\left(e^{-1} d e\right)^{A}{ }_{B}\left(e^{-1} d e\right)_{C}^{B}\left(e^{-1} d e\right)_{A}^{C} \equiv \frac{1}{2} \int\left(e^{-1} d e\right)^{3} \\
\rightarrow \frac{1}{3} \int\left(e^{-1} d e\right)^{3}-\frac{1}{2} \int \varepsilon^{\mu \nu \rho} \partial_{\rho}\left(\phi \partial_{\mu} e_{A}^{\kappa} \partial_{\nu} e_{\kappa}^{A}\right) .
\end{aligned}
$$

The boundary term in Eq. (A.16) transforms according to

$$
\int_{\partial M_{3}} \varepsilon^{i j} \partial_{i} e_{\kappa}^{A} \Gamma_{j \lambda}^{\kappa} e_{A}^{\lambda} \int_{\partial M_{3}} \varepsilon^{i j}\left(\partial_{i} e_{\kappa}^{A} \Gamma_{j \lambda}^{\kappa} e_{A}^{\lambda}\right)+\int_{\partial M_{3}} \varepsilon^{i j}\left(\partial_{i} e_{j}^{A} e_{A}^{l}-\partial_{i} e_{\kappa}^{A} g^{\kappa l}\right) \partial_{l} \phi .
$$

Combining the terms in (A.15), (A.16) and (A.17) one obtains the Weyl transformation of the action $I$ :

$$
I \rightarrow I+\frac{1}{2} \int_{\partial M} \varepsilon^{i j}\left[\Gamma_{\rho i}^{\gamma} g^{\rho k} g_{j \gamma}+\partial_{i} e_{j}^{A} e_{A}^{k}-\partial_{i} e_{\mu}^{A} g^{\mu k} e_{j A}\right] \partial_{k} \phi .
$$

The $\partial_{k} \phi$ terms in (A.18) can be finally simplified to

$$
\int_{\partial M_{3}} \varepsilon^{i j} \partial_{i} e_{j}^{A} e_{A}^{k} \partial_{k} \phi
$$

\section{References}

1. Wen, X.G.: Phys. Rev. B 40, 7387 (1989); Phys. Rev. B 43, 11025 (1991)

2. Fröhlich, J., Studer, U.M.: Incompressible quantum fluids, gauge invariance and current algebra. To appear in the proceedings of Cargèse School of Physics, 1991. Editors G. 't Hooft et al.

3. Polyakov, A.M.: Mod. Phys. Lett. A2, 899 (1987); Khnizhnik, V.G., Polyakov, A.M., Zamolodchikov, A.A.: Mod. Phys. Lett. A3, 819 (1988)

4. David, F.: Mod. Phys. Lett. A3, 1651 (1988); Distler, J., Kawai, H.: Phys. Lett. B 321, 509 (1989)

5. Chamseddine, A.H.: Phys. Lett. B 256, 379 (1991)

6. Alvarez-Gaumé, L., Witten, E.: Nucl. Phys. B 234, 269 (1983)

7. Bardeen W.A., Zumino, B.: Nucl. Phys. B 244, 421 (1984); Alvarez-Gaumé, L., Ginsparg, P.: Ann. Phys. (N.Y.) 161, 423 (1985)

8. Leutwyler, H.: Phys. Lett. 153B, 65 (1985)

9. Jackiw, R.: In Current Algebras and Anomalies. Treimann S.B., Jackiw, R., Zumino, B., Witten E.: (eds.) Singapore: World Scientific 1985, and references therein

10. Di Vecchia, P., Durhuus, B., Petersen, J.L.: Phys. Lett. 144B, 245 (1989)

11. Alvarez-Gaumé, L., Bost, J.-B., Moore, G., Nelson P., Vafa, C.: Phys. Lett. B 178, 105 (1986); Gawędzki, K.: In Non-perturbative Quantum Field Theory. p. 101, editors G 't Hooft et al, New York: Plenum Press 1988

12. Dereli, T., Mukherjee, M., Tucker, R.W.: Class. Quant. Grav. 5, L 21 (1988)

13. Witten, E.: Nucl. Phys. B. 311, 96 (1988); B 323, 113 (1989)

14. Bar-Natan, D., Witten, E.: Commun Math. Phys. 141, 423 (1991)

15. Witten, E.: Commun Math. Phys. 121, 351 (1989)

16. Mavromatos, N., Miramontes, J.: Mod. Phys. Lett. A4, 1847 (1989)

17. Moore, G., Seiberg, N.: Phys. Lett. 220B, 422 (1989); Fröhlich, J., King, C.: Commun. Math. Phys. 126, 167 (1989) 